\title{
Two-timescale stochastic Langevin propagation for classical and quantum optomechanics
}

\author{
M. J. Akram, E. B. Aranas, N. P. Bullier, J. E. Lang, and T. S. Monteiro* \\ Department of Physics and Astronomy, University College London, Gower Street, London WC1E 6BT, United Kingdom
}

(Received 3 August 2018; published 19 December 2018)

\begin{abstract}
Interesting experimental signatures of quantum cavity optomechanics arise because the quantum back-action induces correlations between incident quantum shot noise and the cavity field. While the quantum linear theory of optomechanics (QLT) has provided vital understanding across many experimental platforms, in certain new setups it may be insufficient: analysis in the time domain may be needed, but QLT obtains only spectra in frequency space; and nonlinear behavior may be present. Direct solution of the stochastic equations of motion in time is an alternative, but unfortunately standard methods do not preserve the important optomechanical correlations. We introduce two-timescale stochastic Langevin (T2SL) propagation as an efficient and straightforward method to obtain time traces with the correct correlations. We show that T2SL, in contrast to standard stochastic simulations, can efficiently simulate correlation phenomena such as ponderomotive squeezing and reproduces accurately cavity sideband structures on the scale of the applied quantum noise and even complex features entirely submerged below the quantum shot noise imprecision floor. We investigate nonlinear regimes and find that, where comparison is possible, the method agrees with analytical results obtained with master equations at low temperatures and in perturbative regimes.
\end{abstract}

DOI: 10.1103/PhysRevA.98.063827

\section{INTRODUCTION}

The field of optomechanics offers a rich arena for investigation of quantum effects, at mesoscopic or macroscopic scales, on a wide range of experimental platforms including cantilevers, microtoroids, membranes, and photonic crystals [1-3]. Cavity optomechanics allows manipulation and readout of the states of small mechanical oscillators via interaction with a cavity optical mode which is intrinsically nonlinear. The hugely successful quantum linear theory (QLT) of optomechanics has been used to analyze major experimental milestones; its ease of use and versatility means it is arguably the most widely used analytical tool in cavity optomechanics. But the importance of nonlinear regimes has also been recognizsed [4-8], given possibilities for nonclassical state preparation and quantum nondemolition (QND) measurements. In certain cases, analysis of nonlinearities via perturbative or quantum master equation approaches may be used; but this is not always the case as nonlinearities in recent experiments can be large $[9,10]$, or quantum optomechanical effects are seen at higher temperatures $[11,12]$ where the state space is too large.

We have shown previously that nonlinearities are important in recent experiments involving levitated nanoparticles which exhibit strong nonlinear position coupling to light, with couplings of $g \propto \cos ^{2} k \hat{x}[13,14]$; other optically trapped setups exhibit Gaussian position couplings [15,16]. Further, the dynamics can evolve in time from strongly nonlinear to linear regimes [14], so temporal analysis is useful. New methods of processing and filtering of the optomechanical experimental time trace (prior to Fourier transforming) have been demonstrated experimentally $[17,18]$, which further

\footnotetext{
*t.monteiro@ucl.ac.uk
}

motivates analysis in the time domain. However, QLT does not allow nonlinearity; nor does it yield temporal behaviors as it directly yields power spectral densities (PSDs) in frequency space.

Attention is thus turning to explicit solutions of the underlying nonlinear classical or semiclassical stochastic equations of motion, and these have proved extremely successful for simulation of experiments in cavities $[14,19]$ or other optically trapped setups [18,20], for temporal cooling dynamics as well as asymptotic, steady-state regimes.

Solution of the stochastic equations of motion is in principle straightforward and may be implemented with standard methods and widely available tools [21]. What is not so widely appreciated is that the normal propagation methods fail to preserve the vital important correlations between incoming quantum shot noise $a_{\text {in }}$ and the calculated intracavity field $a(t)$, mediated by the backaction. For fully thermal optomechanical regimes this is not a problem, but otherwise including these correlations correctly is essential. The imprecision quantum-noise-back-action correlations underlie two central signatures of quantum optomechanics: ponderomotive squeezing of the optical field and Raman sideband asymmetry [22-29]. The optimal balance between incoming imprecision noise and the back-action component yields the standard quantum limit (SQL) of force and displacement sensing; squeezing is being investigated as a means to overcome this.

The failure of the standard solutions to preserve noise correlations is not because the Langevin equations replace quantum operators by classical complex functions $\hat{a}(t) \rightarrow a(t)$. These correlations remain relevant even in nonquantum regimes, where their effects are termed noise squashing rather than noise squeezing [30].

The problem is that while the measured signal obtains the output signal $\hat{a}_{\text {out }}(t)$ incorporating correctly all correlations, 
the calculated signal must rebuild them from an input-output relation such as $\hat{a}_{\text {out }}(t)=\hat{a}_{\text {in }}-\sqrt{\kappa} \hat{a}(t)$ for a single-sided cavity. Preserving correlations between the Markovian shot noise $\left[\left\langle\hat{a}_{\text {in }}(t) \hat{a}_{\text {in }}^{\dagger}\left(t^{\prime}\right)\right\rangle=\delta\left(t-t^{\prime}\right)\right]$ and the intracavity dynamics necessitates the complete history of the noise.

In other words, for a measured signal of length $T$, the experimental time trace is sampled on a not too small time step $\Delta t$, so the dimension $N=T / \Delta t$ allows for Fourier transforming, filtering, or another further analysis. In contrast, for the corresponding theoretical spectrum of span $T$, the Wiener increments (stochastic noise) are applied on the computational time step $\delta t \ll \Delta t$, so any trace with the full noise history is so long $N_{\delta t}=T / \delta t \gg N$ that even a simple fast Fourier transform (FFT), let alone more complicated temporal analyses (e.g., [17]), become impractical; one may attempt to sample them: this maintains thermal features but would erase the correlation information.

In the present work we propose and test the two-timescale stochastic Langevin method (T2SL), a temporal propagation technique that reproduces these correlations. The first step is to separate deterministic and stochastic components of the propagation. The simple premise is that a bath applying much stronger (but far fewer) kicks of variance $\sim \Delta t \gg \delta t$ has an equivalent effect. Hence we intersperse deterministic propagation with noise from a (still Gaussian) distribution of higher variance. We can then judiciously pair the $N$ noise contributions to obtain cavity output fields with the correct correlations.

Comparisons with QLT in linear regimes show the method is accurate and robust enough to easily obtain sideband structures down to the scale of the applied quantum shot noise; in fact it reproduces nontrivial dynamical features completely "submerged" in the quantum shot noise floor and corresponding to near-ground-state occupancy. Such features would be considered "beyond the SQL" and would, in experiments, be reported as significant signatures of quantum optomechanics. It is known that such quantum squeezing can be described by linear theory even semiclassically [31]. However, to date, a correlated explicit stochastic demonstration of sidebands "beyond" the SQL has not been demonstrated. It requires only modest, single-desktop computational effort. We then investigate nonlinear regimes and compare with quantum perturbative methods where possible.

In Sec. II we briefly introduce standard optomechanical equations, mainly to define notation, so readers familiar with cavity optomechanics and QLT can move past this section. In Sec. III we introduce T2SL. In Sec. IV we test the accuracy of the correlated T2SL in a broad range of scenarios to reproduce squeezing, including displacement sidebands weak enough to be below the shot noise floor. Thus the significant and surprising finding here is that the quantum limit of weak shot noise is easily achievable (i.e., that the weaker the sideband, the more challenging is the stochastic averaging required to calculate it). We compare with the results of temporal filtering. Such filtering is of course not possible in QLT. We apply T2SL to the nonlinear regime. To test the method we compare with perturbative nonlinear results obtained in [6] but use it to investigate squeezing in the presence of stronger nonlinear coupling, at higher phonon occupancies. Finally in Sec. V we discuss how the method may complement and augment
QLT in the context of quantum optomechanics, and we conclude.

\section{CAVITY OPTOMECHANICS}

The simplest optomechanical systems couple a mechanical oscillator to another oscillator corresponding to the optical mode of a cavity, and the essential physics is well described by the Hamiltonian

$$
\hat{H}=\hbar \Delta \hat{a}^{\dagger} \hat{a}+\left[\frac{\hat{p}^{2}}{2 m}+\frac{1}{2} m \omega_{M}^{2} \hat{x}^{2}\right]+\hat{H}_{\mathrm{int}}+\hat{H}_{\mathrm{diss}} .
$$

In the first term, $\hat{a}\left(\hat{a}^{\dagger}\right)$, represent annihilation (creation) operators of the optical field mode, which is coupled to a mechanical oscillator with displacement operator $\hat{x}=x_{z p f}\left(\hat{b}+\hat{b}^{\dagger}\right)$, where $\hat{b}\left(\hat{b}^{\dagger}\right)$ represent the annihilation (creation) operators of the mechanical mode and $x_{z p f}=\sqrt{\hbar / 2 m \omega_{M}}$. Below we set $\hbar=1$.

$\hat{H}_{\text {int }}$ represents the light matter interaction while $\hat{H}_{\text {diss }}$ represent dissipative processes; for the well-known linear coupling case, $\hat{H}_{\text {int }}=G_{0} \hat{a}^{\dagger} \hat{a} \hat{x}$ where $G_{0}$ is the one-photon coupling strength. To date a linearized analysis considering small fluctuations about the mean, $\hat{a} \rightarrow \bar{\alpha}+\hat{a}(t)$ with effective coupling $\hat{H}_{\text {int }}=g_{1}\left(\hat{a}^{\dagger}+\hat{a}\right) \hat{x}$ where $g_{1} \equiv G_{0} \bar{\alpha}$, has provided a successful bridge between theory and experiment in numerous optomechanics studies. Nonlinear dynamics arising from position squared coupling $H_{\text {int }}=g_{2}\left(\hat{a}^{\dagger}+\hat{a}\right) \hat{x}^{2}$ can also arise. Unfortunately, nonlinearity (whether optical or position squared) has proved difficult to investigate experimentally as it was not previously possible to achieve strong enough coupling strengths; however, new experiments have demonstrated larger or extremely high nonlinear couplings [10,32]. In addition, at higher temperatures, experiments with levitated particles also show $x^{2}$ dynamics [14].

It is straightforward to generalize the dynamics to higher numbers of optical and mechanical modes which are directly or indirectly coupled in the equations of motion and are subjected to corresponding noise baths (e.g., photon shot noise for the optical modes, or incoming phonons for the mechanical modes). We can represent these coupled optical and mechanical modes by a vector $\mathbf{X}(t)=$ $\left(\begin{array}{llllllll}\hat{a}_{1} & \hat{a}_{1}^{\dagger} & \hat{a}_{2} & \hat{a}_{2}^{\dagger} & \cdots & \hat{b}_{1} & \hat{b}_{1}^{\dagger} & \cdots\end{array}\right)^{\top}$, where the $\hat{a}_{j}$ for $j=1,2, \ldots$ are different optical modes, for instance for readout, cooling, or control. Similarly the $\hat{b}_{i}$ represents the mechanical modes (either distinct mechanical oscillators or different degrees of freedom of a single oscillator). The evolution of this $N$-dimensional vector, for any instance of linearized optomechanics, is given by the form

$$
\dot{\mathbf{X}}=\mathbf{A}(t) \mathbf{X}+\sqrt{\boldsymbol{\Gamma}} \zeta(t),
$$

where $\mathbf{A}(t)$ is an $N \times N$ dimensioned drift matrix, which in general (say in modulated optomechanics) can depend explicitly on time, while $\sqrt{\boldsymbol{\Gamma}}=$ diag

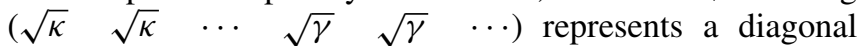
matrix of damping coefficients while $\zeta(t)=\hat{a}_{\mathrm{in}, 1}(t)$ $\hat{a}_{\mathrm{in}, 1}^{\dagger}(t) \quad \cdots \quad \hat{b}_{\mathrm{in}, 1}(t) \quad \hat{b}_{\mathrm{in}, 1}^{\dagger}(t) \quad \ldots{ }^{\top}$ represents the (usually Gaussian) noise baths acting on each mode. The $j$ th element of the vector is $(\mathbf{A X})^{(j)}=\frac{1}{i}\left[\mathbf{X}^{(j)}, \hat{H}\right]-\frac{1}{2}(\mathbf{\Gamma X})^{(j)}$. 
For the simplest case of one optical mode and one mechanical oscillator, the individual equation for the readout (meter) intracavity field (we take $\hat{a}_{1} \equiv \hat{a}$ below) becomes simply

$$
\dot{\hat{a}}=\left(i \Delta-\frac{\kappa}{2}\right) \hat{a}+i g \alpha\left(\hat{b}+\hat{b}^{\dagger}\right)+\sqrt{\kappa} \hat{a}_{\text {in }} .
$$

Solution of Eq. (2) yields the intracavity dynamics. Comparison with experiment requires the further step of obtaining the output mode $\hat{a}_{\text {out }}=\hat{a}_{\text {in }}-\sqrt{\kappa} \hat{a}(t)$ between the imprecision noises $\hat{a}_{\text {in }}$ incident on the cavity and the intracavity field $\hat{a}(t)$. In addition, measurement of the output field by balanced homodyne or heterodyne detection involves the amplification of the signal by beating with an external reference oscillator. For phase $\theta$ one measures $X_{\theta}=\hat{a}(t) e^{-i(\Omega T+\theta)}+\hat{a}^{\dagger}(t) e^{i(\Omega t+\theta)}$ where the heterodyne frequency is $\Omega$ and where $\Omega=0$ for homodyne detection. This corresponds to detection of a fixed optical quadrature (homodyne detection) or a rotating quadrature (heterodyne detection). The power spectral density (PSD) is $S_{X_{\theta} X_{\theta}}(\omega)=\left\langle\left|X_{\theta}(\omega)\right|^{2}\right\rangle$.

For QLT, the PSD is obtained by transforming Eq. (2) into Fourier space and the averaging of the noise (denoted by the \langle\rangle ) by substituting noise correlators. The PSD can also be expressed in terms of the cavity field components:

$S_{X_{\theta} X_{\theta}}(\omega)=S_{\hat{a} \hat{a}^{\dagger}}(\omega)+S_{\hat{a}^{\dagger} \hat{a}}(\omega)+S_{\hat{a}^{\dagger} \hat{a}^{\dagger}}(\omega) e^{2 i \theta}+S_{\hat{a} \hat{a}}(\omega) e^{-2 i \theta}$,

hence the PSD is the sum of an incoherent part and correlations $S_{\text {corr }}=S_{\hat{a}^{\dagger} \hat{a}^{\dagger}}(\omega) e^{2 i \theta}+S_{\hat{a} \hat{a}}(\omega) e^{-2 i \theta}$ (in effect the result of correlations established via the mechanical oscillator between the quadratures of the optical fields).

While the standard linear regime of optomechanics is well understood, new types of dynamics arising from modulation of experimental parameters still motivate the development of new approaches to calculation of the spectra [14,19,33] within the ambit of QLT, which involves a fully quantum solution. However the time trace is not obtained from a QLT solution, which yields only steady state spectral functions. The measured power spectral density (PSD) in contrast is obtained by a Fourier transform (FT) of the experimental time trace, with suitable ensemble averaging.

The corresponding semiclassical equations may be solved in the time domain. Their form is equivalent to Eq. (2), but replacing operators with their expectation values, thus $\hat{a} \rightarrow\langle a\rangle$ and $\hat{b} \rightarrow\langle b\rangle$. This approximation is discussed in optomechanics textbooks (e.g., Chap. 2 of [3]) and is valid when quantum fluctuations are small, thus for reasonably high photon and phonon occupancies. The former is the case in the majority of cavity optomechanics experiments where the cavity photon number $\gg 1$. Numerical tests with QLT show excellent agreement for phonon occupancies down to $n_{m} \sim 10$.

Generalizsing the solution of stochastic equations of motion for the nonlinear case represents a straightforward modification even for multiple interacting modes:

$$
\dot{\mathbf{X}}=\mathbf{A}(\mathbf{X}, t)+\sqrt{\mathbf{\Gamma}} \zeta(t)
$$

In this case, QLT is not valid, but semiclassical solutions in the time domain are possible. The classical equations may be solved by standard packages such as XMDS [21,34].

However, the explicit stochastic propagation of either Eq. (2) or Eq. (4) does not show the nontrivial correlations established in the output mode $\hat{a}_{\text {out }}=\hat{a}_{\text {in }}-\sqrt{\kappa} \hat{a}(t)$ between the imprecision noises $\hat{a}_{\text {in }}$ incident on the cavity and the intracavity field $\hat{a}(t)$ arising from back-action, mediated by the mechanical motion which motivates the T2SL method presented below.

\section{T2SL}

Numerical propagation of Eq. (4) proceeds via an increment:

$$
\mathbf{X}(t+\delta t)-\mathbf{X}(t) \equiv d \mathbf{X}(t)=F(\mathbf{A}, \zeta(t)) .
$$

In general, this is constructed via an increment $F$ which can depend on both deterministic as well as stochastic noise terms. Here we start by adopting rather a propagation method which employs independent increments for the deterministic and stochastic terms, such as the stochastic Runge-Kutta algorithm [35]. For the optomechanics problems where the stochastic components are weighted by a simple constant matrix, convergence is robust, so we may write for our increment $d \mathbf{X}(t)=R(\mathbf{A}) \delta t+d \mathbf{W}(\delta t)$, where $R(\mathbf{A}) \delta t$ denotes, e.g., (deterministic) Runge-Kutta propagation for a time interval $\delta t$, and $d \mathbf{W}(\delta t)$ represents Gaussian noise baths of zero mean and of variance $\sqrt{\delta t}$ for the optical and mechanical modes.

However, the problem still remains that to simulate an experimental trace of duration $T$ the number of propagation steps $N_{T}=T / \delta t \gg 10^{7}$ is extremely large, so preserving the correlations generated by the full history of all these Markovian kicks would still be unfeasible. Hence for T2SL we introduce a much larger time step $\Delta t$ for the noise kicks.

The first stage is deterministic propagation for an interval $\Delta t$ :

$$
\mathbf{X}\left(t+\Delta t^{-}\right)=\int_{t}^{t+\Delta t^{-}} \mathbf{A}(\mathbf{X}, t) d t
$$

where the integral indicates propagation of the coupled equation of motion by some convenient numerical algorithm (taking many small time computational time steps $\delta$ ) and where $t+\Delta t^{-}$denotes the instant just before the noise "kick."

This is followed by application of the Gaussian noise increments:

$$
\mathbf{X}(t+\Delta t)=\mathbf{X}\left(t+\Delta t^{-}\right)+d \mathbf{W}(\Delta t) .
$$

The correlators of the noise bath (Appendix C) are unchanged. This is then repeated, to generate a time series $\left\{\mathbf{X}\left(t_{j}=\right.\right.$ $j \Delta t)\}$ for $j=1,2, \ldots, N_{K}$ from a series of random kicks $d \mathbf{W}^{(j)}(\Delta t)$, where $N_{K}=T / \Delta t \ll T / \delta t$ for a signal of length $T$.

In the optomechanical systems of interest we have, for the $j$ th noise kicks at time $t_{j}$, a vector

$$
\begin{aligned}
& d \mathbf{W}^{(j)}=\left(\begin{array}{lllll}
d W_{1}^{(j)} & d W_{2}^{(j)} & \ldots & d W_{k}^{(j)} & \ldots
\end{array}\right)^{\top} \\
& =\left(\sqrt{\kappa\left(n_{p}+1 / 2\right)} \zeta_{p}^{(j)} \cdots \sqrt{\gamma\left(n_{m}+1 / 2\right)} \zeta_{m}^{(j)} \ldots\right)^{\top},
\end{aligned}
$$

of dimension $l=1,2, \ldots, 2 n_{\text {mod }}$, where $n_{\text {mod }}$ is the number of independent modes (photon and mechanical, so $2 n_{\bmod }=N$ corresponds to the dimension of the drift matrix in Eq. (2), and where the corresponding $\zeta_{p, m}^{(j)}$ (photon and mechanical) 
are $2 n_{\text {mod }}$ Gaussian random numbers drawn from distributions of variance $\sqrt{\Delta t}$. Here, $n_{p}$ and $n_{m}$ are the occupancies of the optical and thermal phonon baths respectively. $n_{p}=0$ corresponds to photon quantum shot noise. We note

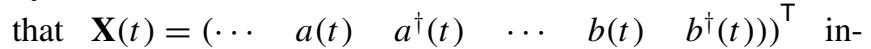
cludes all modes.

We focus on the probe beam/readout mode $a(t)$, which we take to be the lth element of $\mathbf{X}(t)$. The corresponding component in Eq. (7) which propagates the intracavity field of this mode is $a(t=j \Delta t)=a\left(t=j \Delta t^{-}\right)+d W_{l}^{(j)}$. The above is standard stochastic numerics, other than the fact that the noise time step (and its variance) is much larger, $\Delta t \gg \delta t$. Numerical comparisons show that the intracavity field is insensitive to this modification and excellent agreement with QLT is still obtained in linear regimes.

The key step in T2SL is the propagation of the output field. We consider the input-output form for a single-sided cavity, $\hat{a}_{\text {out }}=\hat{a}_{\text {in }}-\sqrt{\kappa} \hat{a}(t)$, but our method is easily adapted to other cases. This includes the case of nonideal detection and losses in the optical system, modeled as a single loss port in a recent experimental study [26]. We propose the following stochastic increments for the correlated output field:

$$
a_{\text {out }}\left(t_{j}=j \Delta t\right)=\mathcal{C} d W_{l}^{(j)}-\sqrt{\kappa}\left[a\left(j \Delta t^{-}\right)+d W_{l}^{(j)}\right],
$$

where $\mathcal{C}$ is a constant. The second term (in square brackets) is simply the update to the intracavity field, while the first term is the imprecision noise. We choose the normalization $\mathcal{C}$ such that the time series $\left\{\mathcal{C} d W_{l}^{(j)}\left(t_{j}\right)\right\}$ gives a flat noise spectrum of height $n_{p}+1 / 2$ (and specifically height $=1 / 2$ for quantum shot noise for which $\left.n_{p}=0\right)$. Here we took $\mathcal{C}=1 /(\sqrt{\kappa} \Delta t)$ but this can be adjusted for alternative implementations, e.g., depending on the FFT normalizations. The main point is to set $\mathcal{C}$ such that if $a_{\text {out }}\left(t_{j}\right)=\mathcal{C} d W_{l}^{(j)}$, the required white noise floor level is obtained, and to ensure that the same noise kick $d W_{l}^{(j)}$ is used in both terms in Eq. (9). Clearly the term $a\left(t=j \Delta t^{-}\right)$contains the past history of kicks $1,2, \ldots, j-$ 1 which drive the dynamics and back-action. It carries all the narrowband features that interfere with the imprecision floor.

However, we show below that this pairing of kicks in Eq. (9) preserves the important correlations and so obtains spectral features of both classical and even certain important regimes of quantum optomechanics.

\section{APPLICATIONS OF T2SL}

We now test and consider applications of T2SL to a variety of regimes, including comparisons with spectra in quantum regimes.

\section{A. Linear: Quantum optical squeezing}

The resultant explicit stochastic numerics are in excellent agreement with results obtained from analytical quantum noise spectra in linearized regimes. Figure 1(a) compares the stochastic model and QLT analytical spectra corresponding to recent experiments [26] on ponderomotive squeezing. The comparison shows these regimes of phonon occupancies of tens of quanta are easily calculated stochastically with modest averaging of a few hundred trajectories, taking minutes of CPU time on a desktop.

\section{B. Linear: Modulated quantum optomechanics}

Figure 1(b) presents a more much more challenging test of T2SL: a regime modeling a levitated nanoparticle in a hybrid optical cavity Paul trap in the quantum back-action limit. The trapping potential introduces a temporal modulation which introduces nonstationary components in the PSD calculations and makes even the QLT much more complicated. The QLT theory was developed in $[19,33]$ but therein was only compared with stochastics in thermal regimes. Further details are in Appendix A.

Here we are able to compare the modulated QLT with T2SL calculations. In back-action dominated regimes, squeezing can lead to complicated sideband behavior completely immersed below the quantum imprecision floor. These very small structures, on the order of a single quantum and comparable to the noise, are more challenging to obtain. Nevertheless with about 1000 trajectory averaging, a modest calculation yields near exact agreement.

\section{Temporal filtering of correlated signals}

Figure 2 exemplifies the advantages of enabling direct manipulation of temporal traces prior to the FFTs which yield PSDs or other types of spectra. QLT does not allow this as experimental comparison is only possible in frequency space, yet such methods are proving useful in experiments [16,17]. In particular [17] demonstrated a temporal technique to restore homodyne-like features arising from correlations which are eliminated in heterodyne detection. In [17] a time filter was applied to the experimental time trace; then an FFT enabled comparison with the appropriate QLT generated components.

Here we show that we can apply the filter directly to the calculated T2SL trace. This means that filtering protocols can be perfected and tested easily without the need for experiments in squeezing regimes which are laborious and difficult. It would be interesting to apply T2SL to other proposals such as synodyne detection [36,37], which involves two heterodyne reference signals, possibly in combination with filtering. It may also be applied to recover nonstationary components in such spectra or modulated optomechanics.

\section{Nonlinear: Quantum optical squeezing}

It is then straightforward to extend the method to systems with nonlinearities, both optical and position squared; in fact any other type of nonlinearity can thus be simulated and the method may also be applied to other general situations including dissipatively coupled systems. In Fig. 3 PSDs showing both squeezing as well as the effects on nonlinearity are shown. In Fig. 3(a) the $\hat{x}^{2}$ nonlinearity appears in the form of mechanical sidebands at both $\omega=0$ and $\omega=2 \omega_{M}$. The central $\omega=0$ detected peak is far stronger; this is a straightforward effect of the cavity filtering since the calculations corresponded to sideband-resolved regimes with $\omega_{M} \gtrsim \kappa / 2$ so the $2 \omega_{M}$ is strongly suppressed; but it indicates the advantages of detecting weak nonlinearities using the (not so well studied) central DC peak, using careful balanced detection. 
(a) homodyne detection of quantum squeezing

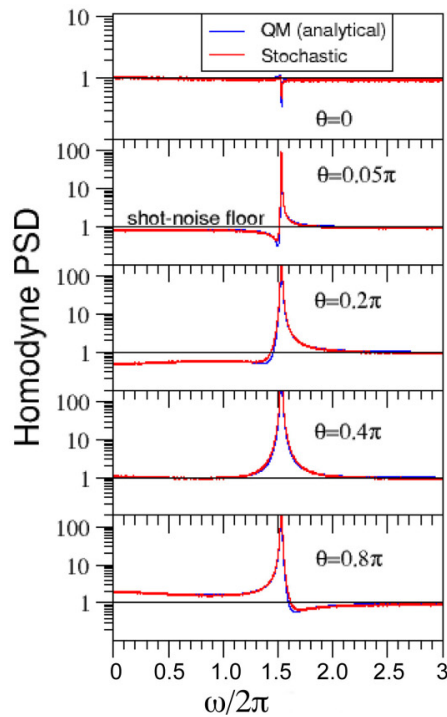

(b) modulated optomechanics

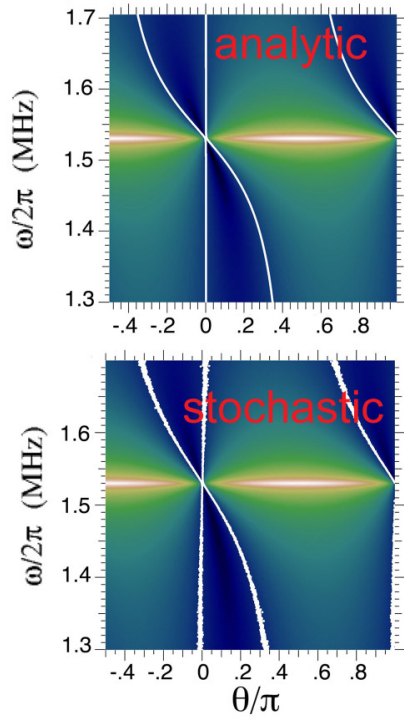

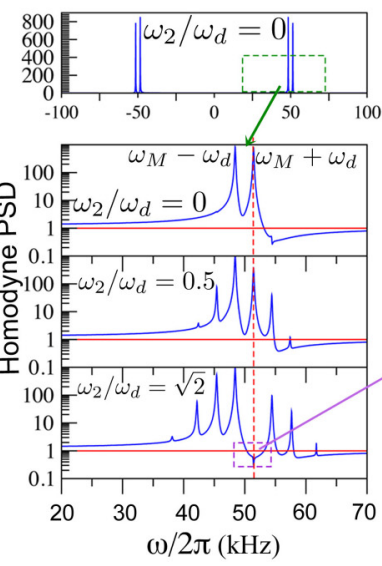

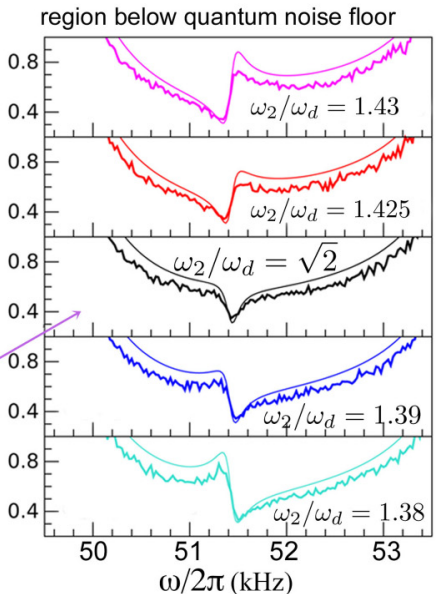

FIG. 1. (a) T2SL reproduces accurately quantum ponderomotive squeezing effects. Comparison with analytical expressions of quantum linearized theory of optomechanics (QLT) is excellent. The presence of correlations $S_{\text {corr }}(\omega)$ between optical field quadratures dependent on the homodyne phase angle result in PSD values below the quantum noise floor. Parameters and results are similar to experimentally observed behavior in quantum squeezing regimes seen in [26], using both a damping and readout optical beams. Left panels illustrate cuts through the color map. The shot noise floor $\equiv 1$. The color map is in a logarithmic scale where white lines denote the shot noise floor and dark blue indicates noise below the shot noise floor. (b) T2SL, surprisingly, perfectly reproduces sideband structures on the scale of the quantum shot noise kicks. The figure illustrates an example with complex sideband structure completely below the quantum shot noise floor. In levitated experiments with hybrid electro-optical traps both the optomechanical coupling $g_{1}(t)=2 \bar{g}_{1} \sin \omega_{d} t$ and the mechanical frequency $\omega_{M}(t)=\bar{\omega}_{M}+2 \omega_{2} \cos \left(2 \omega_{d} t\right)$ are subject to slow modulations $\omega_{d} \ll \omega_{M}$, resulting in split-sideband peaks, split by $2 \omega_{d}$ at $\pm \omega=\omega_{M} \pm \omega_{d}$. The QLT for this case was developed in [19,33]: with increasing $\omega_{2} / \omega_{d}$, the $\omega_{M}+\omega_{d}$ peak is suppressed, then reappears. In squeezing regimes, this second peak is fully submerged below the quantum noise floor: the phase change around the suppression point is well described by T2SL; shot noise $\equiv 1$ so features in the right panels are all below this. Parameters are listed in Appendix D.

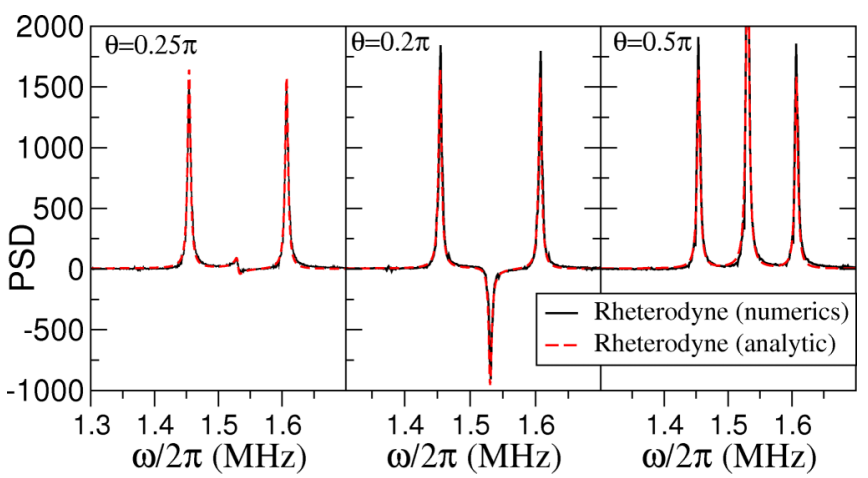

FIG. 2. Illustration of the use of filtering in the time domain. T2SL provides a correlated time trace, thus allowing temporal processing of the calculated heterodyne or homodyne signal, unlike quantum linearized theory of optomechanics (QLT) which directly returns spectra in the frequency domain. Ths figure illustrates the temporal filtering technique termed r-heterodyne, developed and experimentally tested in [17], which extracts homodyne features from a heterodyne-detected spectrum using a temporal filter function. A comparison between the filtered T2SL time trace and the predicted spectrum $S_{\hat{a}^{\dagger} \hat{a}^{\dagger}}(\Omega+\omega)+S_{\hat{a}^{\dagger} \hat{a}}(\Omega-\omega)+S_{\hat{a}^{\dagger} \hat{a}^{\dagger}}(\omega) e^{2 i \theta}+S_{\hat{a} \hat{a}}(\omega) e^{-2 i \theta}$ shows excellent agreement, showing the usefulness of T2SL correlated time traces for allowing the development of new temporal filtering and signal processing methods. Parameters are the same as in Fig. 1(a).
The clearest advantage is that the effects of squeezing are strong near $\omega=0$ and, for a system with $g_{1} \sim g_{2}$, the SQL can be overcome at the mechanical frequency itself, unlike the case for pure linear coupling. All the simulations in Fig. 3 average over 500-1000 stochastic realizations.

\section{E. Nonlinear: Comparison with quantum perturbative methods}

In Fig. 4 we compare T2SL for a pure nonlinear (position squared) coupling near zero temperature with analytical expressions obtained perturbatively in [6]. This was the most challenging case numerically as the coupling is extremely weak and involves an extreme quantum regime of low phonon occupancy. It required tens of thousands of realizations. Although not the most appropriate regime for T2SL, it indicates that position-squared nonlinearities are also adequately captured by T2SL.

\section{DISCUSSION: T2SL FOR QUANTUM OPTOMECHANICS}

We have shown that T2SL accurately reproduces quantum ponderomtive squeezing regimes. However, we note that it does not obtain Raman sideband asymmetries, another key experimental signature of the quantum optomechanical regime which can arise from the quantum back-action [38,39]. 


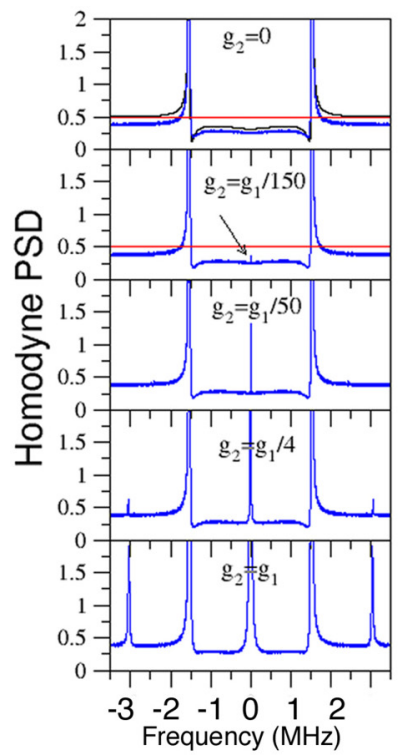

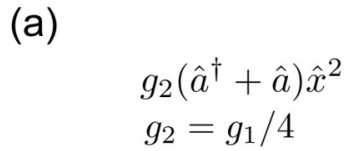

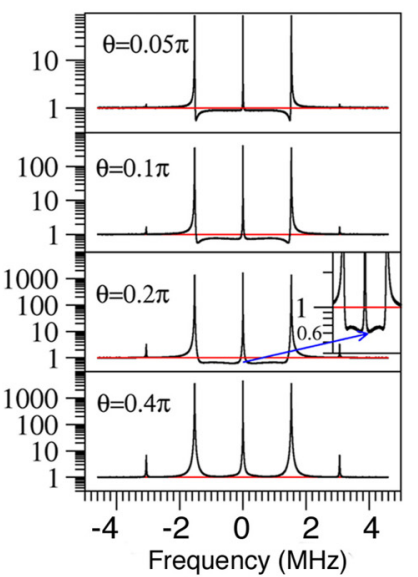

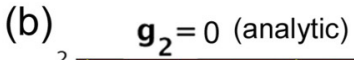

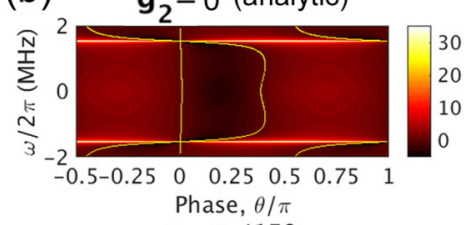$$
\mathbf{g}_{2}=\mathbf{g}_{1} / \mathbf{1 5 0}
$$

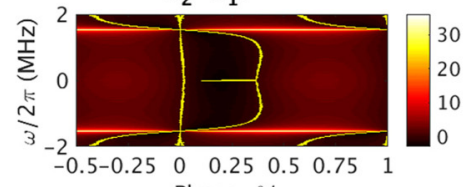

Phase, $\theta / \pi$

$\mathrm{g}_{2}=\mathrm{g}_{1} / 4$

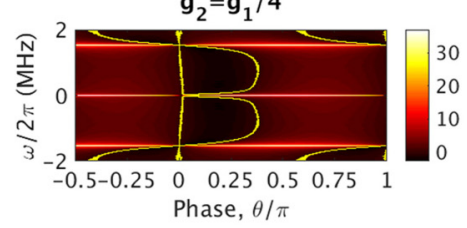

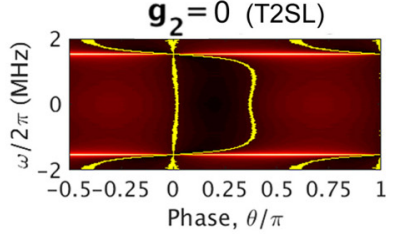

Phase, $\theta / \pi$

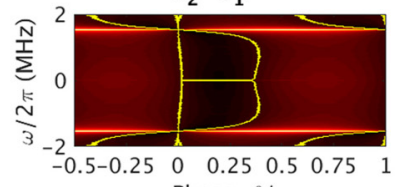

Phase, $\theta / \pi$

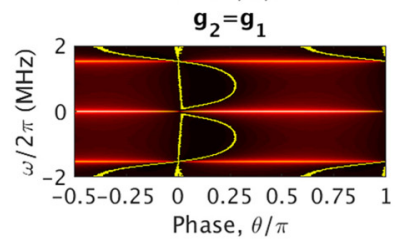

FIG. 3. T2SL for nonlinear quantum optomechanics. T2SL is used in a regime combining linear and position-squared nonlinearity using the parameters of Ref. [26], where the frequency of the mechanical oscillator is $\omega_{M} / 2 \pi=1.53 \mathrm{MHz}$. (a) A set of homodyne PSDs (for $\theta=0.05 \pi$ in the left panels; as a function of $\theta$ in the right panels) for a system with optomechanical interaction $H_{\text {int }}=g_{1}\left(\hat{a}^{\dagger}+\hat{a}\right) \hat{x}+g_{2}\left(\hat{a}^{\dagger}+\hat{a}\right) \hat{x}^{2}$ with parameter similar to Fig. 1(a) apart from the additional nonlinear $g_{2}$ term. The top panel, for $g_{2}=0$, shows a comparison with QLT. It shows the interaction of ponderomotive squeezing with nonlinearity with increasing $g_{2}$. Note that the central nonlinear peak (left panels) for weaker nonlinearity $g_{2} \lesssim 10^{-2} g_{1}$ can be fully submerged below the quantum shot noise floor, indicated by red line. (b) Corresponding color maps where black maps the region below quantum shot noise for different values of nonlinear position-squared coupling. Remaining parameters are the same as in Fig. 1(a).

It is useful here to divide optomechanical quantum signatures which arise from correlations between the quantum shot noise and the intracavity field into two classes: (i) those that are sensitive to the commutation relation $\left\langle\left[\hat{a}_{\text {in }}(t), \hat{a}_{\text {in }}^{\dagger}\left(t^{\prime}\right)\right]\right\rangle=$

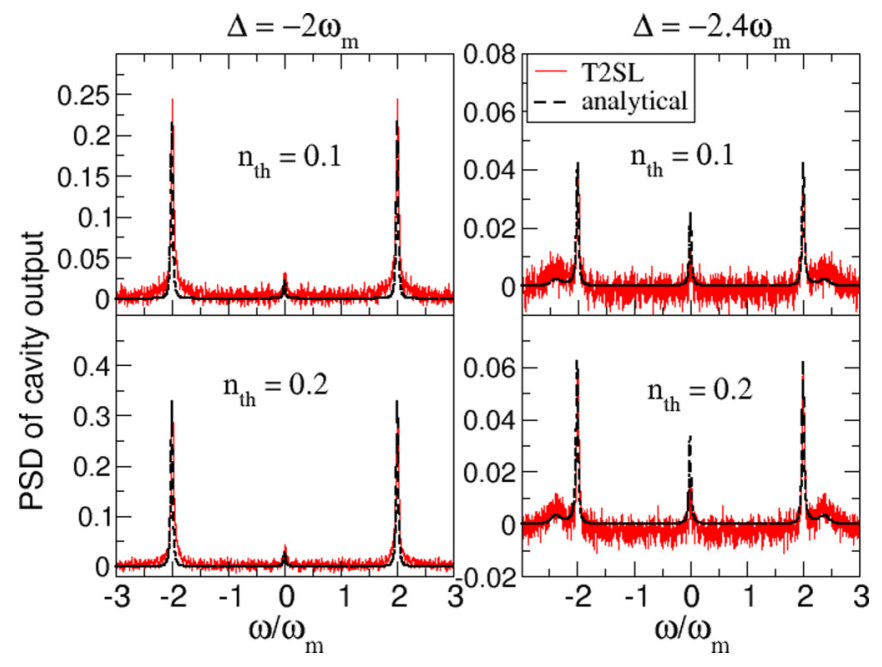

FIG. 4. T2SL for nonlinear quantum optomechanics. T2SL may be employed in regimes of extremely low thermal occupancies $n_{\text {th }} \lesssim$ 1 of the mechanical oscillator. Here we have pure position-squared nonlinearity (linear coupling $g_{1}=0$ ) and optomechanical interaction $H_{\text {int }}=g_{2}\left(\hat{a}+\hat{a}^{\dagger}\right) \hat{x}^{2}$ and bath temperatures $T_{B}=0$, so it is possible to compare with analytical perturbative results [6] (black line). The figure shows the PSD of the cavity output and compares with Eq. (7) of [6] for two values of detuning. Remaining parameters are listed in Appendix D. $\delta\left(t-t^{\prime}\right)$ (this includes Raman sideband asymmetry) and (ii) those that are insensitive and yield the same calculated spectra if one assumes $\left\langle\hat{a}_{\text {in }}(t) \hat{a}_{\text {in }}^{\dagger}\left(t^{\prime}\right)\right\rangle=\left\langle\hat{a}_{\text {in }}^{\dagger}(t) \hat{a}_{\text {in }}\left(t^{\prime}\right)\right\rangle=1 / 2 \delta\left(t-t^{\prime}\right)$ (these include ponderomotive squeezing and quantum optical correlations such as those obtained by homodyne detection or cross-correlation spectra). One might argue that only the former are "real" quantum optomechanical signatures while the latter are classical phenomena. However, the insensitivity is rather a result of the symmetrization of the detected spectra; hence ponderomotive squeezing is an important signature of quantum optomechanics.

Regardless of such classification, optical quantum squeezing is at the heart of current schemes to achieve and overcome the SQL in optomechanical displacement sensing. Although there is currently much interest in the relation between asymmetry in the mechanical sideband spectrum vis-à-vis the cavity output spectrum [38,39] for different detection scenarios, here there is no such ambiguity: the mechanical spectrum is always symmetric for these Langevin simulations of quantum squeezing. Nevertheless, the total sideband area remains correct so the calculations are still useful for sensing of displacement and forces as well as thermometry. Hence T2SL offers a quite straightforward approach to investigate these important regimes for both quantum noise squeezing and classical noise squashing.

\section{CONCLUSION}

We show that by means of a purely classical calculation using T2SL, one can accurately reproduce optomechanical spectra below the standard quantum limit (SQL) in 
narrowband measurements where the homodyne measurement falls below the shot noise imprecision floor. We have investigated regimes with simultaneous linear and nonlinear couplings. Even in linear regimes, T2SL complements QLT since the method generates traces in the time domain. It can also be employed in complex linear regimes (multimode optomechanics, modulated optomechanics) as an independent check of QLT. The advantage is that T2SL can then be employed to explore regimes beyond the scope of the usual linear analysis and temporal filtering.

\section{ACKNOWLEDGMENTS}

N.B. acknowledges support from the Engineering and Physical Sciences Research Council (Grant No.
EP/L015242/). M.J.A. is thankful for support via a StocklinSelmoni studentship.

\section{APPENDIX A: OPTOMECHANICAL LANGEVIN EQUATIONS}

The dynamics of a general, $n$-mode optomechanical system is described by $2 n$ coupled equations of motion which, in matrix form, can be expressed as $\dot{\mathbf{X}}=\mathbf{A}(t) \mathbf{X}+\sqrt{\boldsymbol{\Gamma}} \zeta(t)$. For the simplest case where $\mathbf{X}=\left(\begin{array}{llll}\hat{a} & \hat{a}^{\dagger} & \hat{b} & \hat{b}^{\dagger}\end{array}\right)^{\top}$, where $\hat{a}, \hat{b}$ are optical and mechanical modes, the linearized, coupled Langevin equations in time are explicitly given by

$$
\left(\begin{array}{c}
\dot{\hat{a}}(t) \\
\dot{\hat{a}}^{\dagger}(t) \\
\hat{\hat{b}}(t) \\
\hat{b}^{\dagger}(t)
\end{array}\right)=\left(\begin{array}{cccc}
i \Delta(t)-\kappa(t) / 2 & 0 & i g(t) & i g(t) \\
0 & -i \Delta(t)-\kappa(t) / 2 & -i g(t) & -i g(t) \\
i g(t) & i g(t) & -i \omega_{M}(t)-\gamma(t) / 2 & 0 \\
-i g(t) & -i g(t) & 0 & i \omega_{M}(t)-\gamma(t) / 2
\end{array}\right)\left(\begin{array}{c}
\hat{a}(t) \\
\hat{a}^{\dagger}(t) \\
\hat{b}(t) \\
\hat{b}^{\dagger}(t)
\end{array}\right)+\left(\begin{array}{c}
\sqrt{\kappa} \hat{a}_{\mathrm{in}}(t) \\
\sqrt{\kappa} \hat{a}_{\mathrm{in}}^{\dagger}(t) \\
\sqrt{\gamma} \hat{b}_{\mathrm{in}}(t) \\
\sqrt{\gamma} \hat{b}_{\mathrm{in}}^{\dagger}(t)
\end{array}\right),
$$

where in general the cavity detuning $\Delta$, mechanical frequency $\omega_{\mathrm{M}}$, optomechanical coupling $g$, and the damping rates $\kappa$ and $\gamma$ can be time dependent. Otherwise, the analytical solution of Eq. (A1) is straightforward in frequency space. The position $x=\left(\hat{b}+\hat{b}^{\dagger}\right) / \sqrt{2}$ and optical amplitude quadrature $y=(\hat{a}+$ $\left.\hat{a}^{\dagger}\right) / \sqrt{2}$ are

$$
\begin{aligned}
& x(\omega)=i g \mu(\omega) y(\omega)+\sqrt{\gamma} \hat{X}_{\mathrm{th}}(\omega), \\
& y(\omega)=\operatorname{ig\eta }(\omega) x(\omega)+\sqrt{\kappa} \hat{Y}_{\mathrm{in}}(\omega),
\end{aligned}
$$

where the input noises are

$$
\begin{aligned}
& \hat{X}_{\text {th }}(\omega)=\chi_{M}(\omega) \hat{b}_{\text {in }}(\omega)+\chi_{M}^{*}(-\omega) \hat{b}_{\text {in }}^{\dagger}(\omega), \\
& \hat{Y}_{\text {in }}(\omega)=\chi_{O}(\omega) \hat{a}_{\text {in }}(\omega)+\chi_{O}^{*}(-\omega) \hat{a}_{\text {in }}^{\dagger}(\omega),
\end{aligned}
$$

and where the mechanical and optical susceptibilities are

$$
\begin{aligned}
& \mu(\omega)=\chi_{M}(\omega)-\chi_{M}^{*}(-\omega), \\
& \eta(\omega)=\chi_{O}(\omega)-\chi_{O}^{*}(-\omega),
\end{aligned}
$$

with

$$
\begin{gathered}
\chi_{M}(\omega)=\left[-i\left(\omega-\omega_{M}\right)+\gamma / 2\right]^{-1}, \\
\chi_{O}(\omega)=[-i(\omega+\Delta)+\kappa / 2]^{-1} .
\end{gathered}
$$

Note that simultaneously solving Eqs. (A2) and (A3) leads to closed form solutions, which is not the case when we consider time-dependent couplings, such as in a doubly modulated optomechanical system.

\section{Doubly modulated optomechanics}

We consider the slow modulations

$$
\begin{aligned}
g(t) & =2 \bar{g} \sin \omega_{d} t, \\
\omega_{M}(t) & =\bar{\omega}_{M}+2 \omega_{2} \cos 2 \omega_{d} t,
\end{aligned}
$$

where $\omega_{d} \ll \omega_{M}$. Such a doubly modulated model explains the split-sideband spectra from hybrid electro-optical trap experiments [33]. Substituting Eqs. (A10) and (A11) in Eq. (A1), while keeping other parameters constant, we obtain in frequency space

$$
\begin{aligned}
x(\omega)= & \bar{g} \mu(\omega)\left[y\left(\omega+\omega_{d}\right)-y\left(\omega-\omega_{d}\right)\right] \\
& +\sqrt{\gamma} \hat{X}_{\mathrm{th}}(\omega)-i \omega_{2} \mathcal{G}(\omega), \\
y(\omega)= & \bar{g} \eta(\omega)\left[x\left(\omega+\omega_{d}\right)-x\left(\omega-\omega_{d}\right)\right] \\
& +\sqrt{\kappa} \hat{Y}_{\mathrm{in}}(\omega) .
\end{aligned}
$$

We see in Eq. (A13) that the main effect of a modulated $g(t)$ is to couple the optical field to the shifted position operator, while a modulated $\omega_{M}(t)$ introduces a correction term $\mathcal{G}(\omega)=\chi_{\mathrm{M}}(\omega)\left[\hat{b}\left(\omega+2 \omega_{d}\right)+\hat{b}\left(\omega-2 \omega_{d}\right)\right]-$ H.c. Ultimately, the $\mathcal{G}(\omega)$ corrections conspire such that the cross-correlation terms of the shifted spectrum $\left\langle\left|x\left(\omega+\omega_{d}\right)-x\left(\omega-\omega_{d}\right)\right|\right\rangle^{2}$ result in destructive interference at $\omega_{M}+\omega_{d}$ when $\omega_{2} \approx \sqrt{2} \omega_{d}$. In the quantum regime, this leads to subshot noise dynamics observed in Fig. 1(b) of the main paper. It is straightforward to generalize to the case where there are two optical modes: one for control, one for readout (probe beam) [19]. 


\section{APPENDIX B: POWER SPECTRAL DENSITY AND THE WIENER-KHINCHIN THEOREM}

The power spectral density (PSD) gives the distribution of power over frequency of a continuous, noisy signal. In optomechanical experiments, the area under the PSD curve of the probe signal is proportional to the temperature of the mechanical oscillator, thus making the PSD a main quantity of interest. The Wiener-Khinchin theorem guarantees that the PSD can be calculated by taking the Fourier transform of the autocorrelation function:

$$
\lim _{T \rightarrow \infty}\left\langle\tilde{u}(\omega)[\tilde{v}(\omega)]^{\dagger}\right\rangle=S_{u v^{\dagger}}(\omega)
$$

where $\quad \tilde{u}(\omega)=\frac{1}{\sqrt{T}} \int_{0}^{T} d t e^{i \omega t} \hat{u}(t) \quad$ and $\quad[\tilde{v}(\omega)]^{\dagger}=\frac{1}{\sqrt{T}}$ $\int_{0}^{T} d t e^{-i \omega t}[\hat{v}(t)]^{\dagger}$ are the gated Fourier transforms of any two operators, and

$$
\begin{aligned}
S_{u v^{\dagger}}(\omega) & =\int_{-\infty}^{+\infty} d t \int_{-\infty}^{+\infty} d \tau e^{i \omega \tau}\left\langle\hat{u}(t+\tau)[\hat{v}(t)]^{\dagger}\right\rangle \\
& =\int_{-\infty}^{+\infty} d \tau e^{i \omega \tau}\left\langle\hat{u}(\tau)[\hat{v}(0)]^{\dagger}\right\rangle,
\end{aligned}
$$

where we assumed in the second line a stationary signal, i.e., we can simply set $t=0$.

\section{R-heterodyne detection of correlations}

For cavity experiments the measureable quantity is in fact not the intracavity field but the cavity output:

$$
\hat{a}_{\text {out }}(\omega)=\hat{a}_{\text {in }}(\omega)+\sqrt{\kappa} \hat{a}(\omega),
$$

where $\hat{a}(\omega)$ consists of the cavity-filtered shot noise, thermal noise from the mechanical mode, and the back-action noise due to optical measurement of the mechanical motion. The interference between the incoming noise $\hat{a}_{\text {in }}(\omega)$ and the cavityfiltered shot noise together form the imprecision noise floor at half a quanta, on which the thermal noise of the mechanical oscillator sits. The imprecision noise is constant throughout the full bandwidth but destructive interference with the optical back-action noise can bring down the noise below the Heisenberg limit, leading to ponderomotive squeezing.

The choice of detection method is crucial in revealing this mechanically mediated optical squeezing. Heterodyne detection combines the bright field of a beam, called the local oscillator (LO), with the cavity output so as to be sensitive only to the linear order of the cavity output:

$$
Y_{\text {het }}(t)=\hat{a}_{\text {out }}(t) e^{-i(\theta+\Omega t)}+\hat{a}_{\text {out }}^{\dagger}(t) e^{i(\theta+\Omega t)},
$$

where $\theta$ is the LO phase, and $\Omega$ is its frequency. Setting $\Omega=0$ gives the homodyne signal:

$$
Y_{\text {hom }}(t)=\hat{a}_{\text {out }}(t) e^{-i \theta}+\hat{a}_{\text {out }}^{\dagger}(t) e^{i \theta} \text {. }
$$

Tuning $\theta$ allows us to measure different quadratures of the cavity output, with $\theta=0(\theta=\pi / 2)$ probing the amplitude (phase) quadrature.

Meanwhile, using Eq. (B1), we derive the heterodyne and homodyne PSDs:

$$
\begin{aligned}
& S_{Y_{\text {het }}}(\omega)=S_{\hat{a} \hat{a}^{\dagger}}(\omega+\Omega)+S_{\hat{a}^{\dagger} \hat{a}}(\omega-\Omega), \\
& S_{Y_{\text {hom }}}(\omega)=S_{\hat{a} \hat{a}^{\dagger}}(\omega)+S_{\hat{a}^{\dagger} \hat{a}}(\omega)+e^{-2 i \theta} S_{\hat{a} \hat{a}}(\omega)+e^{2 i \theta} S_{\hat{a}^{\dagger} \hat{a}^{\dagger}}(\omega) .
\end{aligned}
$$

We see that Eq. (B6) does not converge to Eq. (B7) in the same way that Eq. (B4) converges to Eq. (B5) when we take the limit $\Omega \rightarrow 0$. The terms $S_{\hat{a} \hat{a}}(\omega)$ and $S_{\hat{a}^{\dagger} \hat{a}^{\dagger}}(\omega)$ where ponderomotive squeezing originates are lost.

This loss of correlations can be traced back in the time domain, when we calculate the autocorrelation function of a nonstationary signal such as $Y_{\text {het }}(t)$. Substituting Eq. (B4) in Eq. (B2), we obtain

$$
\begin{aligned}
S_{Y_{\mathrm{het}}}(\omega)= & S_{\hat{a} \hat{a}^{\dagger}}(\omega+\Omega)+S_{\hat{a}^{\dagger} \hat{a}}(\omega-\Omega) \\
& +\int_{-\infty}^{+\infty} d t S_{\mathrm{corr}}(\omega, t),
\end{aligned}
$$

where $S_{\text {corr }}(\omega, t)=S_{\hat{a} \hat{a}}(\omega) e^{2 i(\theta+\Omega t)}+S_{\hat{a}^{\dagger} \hat{a}^{\dagger}}(\omega) e^{-2 i(\theta+\Omega t)}$. The correlations acquire a time dependence that gets averaged out due to the continuous nature of the measurement. The point of r-heterodyning is to convolute the heterodyne signal with an appropriate filter function so that $S_{\text {corr }}(\omega, t)$ may be recovered, thereby exposing optical squeezing.

\section{APPENDIX C: HOMODYNE DETECTION OF PONDEROMOTIVE SQUEEZING: INSENSITIVITY TO NONCOMMUTATIVITY OF OPERATORS}

In the main paper, we have classified quantum signatures depending on whether or not they are sensitive to the quantum noise correlators

$$
\begin{aligned}
& \left\langle\hat{a}_{\text {in }}(t) \hat{a}_{\text {in }}^{\dagger}(0)\right\rangle=\delta(t), \quad\left\langle\hat{a}_{\text {in }}^{\dagger}(t) \hat{a}_{\text {in }}(0)\right\rangle=0, \\
& \left\langle\hat{b}_{\text {in }}(t) \hat{b}_{\text {in }}^{\dagger}(0)\right\rangle=(\bar{n}+1) \delta(t), \quad\left\langle\hat{b}_{\text {in }}^{\dagger}(t) \hat{b}_{\text {in }}(0)\right\rangle=\bar{n} \delta(t),
\end{aligned}
$$

where the mean phonon number $\bar{n}=\left[e^{\hbar \omega_{m} / k_{B} T_{b}}-1\right]^{-1}$, and $T_{b}$ is temperature of the mechanical bath [40]. We show here that the homodyne detection of ponderomotive squeezing is insensitive to noncommutativity of operators, i.e., we can classically simulate quantum squeezing by assuming instead the noise correlators

$$
\begin{aligned}
\left\langle\left\langle\hat{a}_{\text {in }}(t) \hat{a}_{\text {in }}^{\dagger}(0)\right\rangle\right. & =\left\langle\hat{a}_{\text {in }}^{\dagger}(t) \hat{a}_{\text {in }}(0)\right\rangle=\frac{1}{2} \delta(t), \\
\left\langle\hat{b}_{\text {in }}(t) \hat{b}_{\text {in }}^{\dagger}(0)\right\rangle & =\left\langle\hat{b}_{\text {in }}^{\dagger}(t) \hat{b}_{\text {in }}(0)\right\rangle=\left(\bar{n}+\frac{1}{2}\right) \delta(t) .
\end{aligned}
$$


Let us write Eq. (B3) as a function of the vacuum inputs of the optical and mechanical oscillators

$$
\hat{a}_{\text {out }}(\omega)=A_{1}(\omega) \hat{a}_{\text {in }}(\omega)+A_{2}(\omega) \hat{a}_{\text {in }}^{\dagger}+A_{3}(\omega) \hat{b}_{\text {in }}(\omega)+A_{4}(\omega) \hat{b}_{\text {in }}^{\dagger}(\omega),
$$

where $A_{1}(\omega), A_{2}(\omega), A_{3}(\omega)$, and $A_{4}(\omega)$ are complex functions. Using the classical noise correlators we obtain

$$
\begin{aligned}
S_{\hat{a^{\dagger}}}(\omega) & =\frac{1}{2}\left|A_{1}(\omega)\right|^{2}+\frac{1}{2}\left|A_{2}(\omega)\right|^{2}+\left|A_{3}(\omega)\right|^{2}\left(\bar{n}+\frac{1}{2}\right)+\left|A_{4}(\omega)\right|^{2}\left(\bar{n}+\frac{1}{2}\right), \\
S_{\hat{a}^{\dagger} \hat{a}}(\omega) & =\frac{1}{2}\left|A_{1}(-\omega)\right|^{2}+\frac{1}{2}\left|A_{2}(-\omega)\right|^{2}+\left|A_{3}(-\omega)\right|^{2}\left(\bar{n}+\frac{1}{2}\right)+\left|A_{4}(-\omega)\right|^{2}\left(\bar{n}+\frac{1}{2}\right), \\
S_{\hat{a} \hat{a}}(\omega) & =\frac{1}{2} A_{1}(\omega) A_{2}(-\omega)+\frac{1}{2} A_{1}(-\omega) A_{2}(\omega)+A_{3}(\omega) A_{4}(-\omega)\left(\bar{n}+\frac{1}{2}\right)+A_{3}(-\omega) A_{4}(\omega)\left(\bar{n}+\frac{1}{2}\right), \\
S_{\hat{a}^{\dagger} \hat{a}^{\dagger}}(\omega) & =\frac{1}{2} A_{1}^{*}(-\omega) A_{2}^{*}(\omega)+\frac{1}{2} A_{1}^{*}(\omega) A_{2}^{*}(-\omega)+A_{3}^{*}(\omega) A_{4}^{*}(-\omega)\left(\bar{n}+\frac{1}{2}\right)+A_{3}^{*}(-\omega) A_{4}^{*}(\omega)\left(\bar{n}+\frac{1}{2}\right) .
\end{aligned}
$$

Meanwhile, using the quantum noise correlators (C1), we have

$$
\begin{aligned}
S_{\hat{a} \hat{a}^{\dagger}}(\omega)= & \left|A_{1}(\omega)\right|^{2}+\left|A_{3}(\omega)\right|^{2}(\bar{n}+1)+\left|A_{4}(\omega)\right|^{2} \bar{n}, \quad \text { (C8) } \\
S_{\hat{a}^{\dagger} \hat{a}}(\omega)= & \left|A_{2}(-\omega)\right|^{2}+\left|A_{3}(-\omega)\right|^{2} \bar{n}+\left|A_{4}(-\omega)\right|^{2}(\bar{n}+1), \\
S_{\hat{a} \hat{a}}(\omega)= & A_{1}(\omega) A_{2}(-\omega)+A_{3}(\omega) A_{4}(-\omega)(\bar{n}+1) \\
& +A_{3}(-\omega) A_{4}(\omega) \bar{n}, \\
S_{\hat{a}^{\dagger} \hat{a}^{\dagger}}(\omega)= & A_{1}^{*}(\omega) A_{2}^{*}(-\omega)+A_{3}^{*}(\omega) A_{4}^{*}(-\omega)(\bar{n}+1) \\
& +A_{3}^{*}(-\omega) A_{4}^{*}(\omega) \bar{n} .
\end{aligned}
$$

Finally, in Eq. (B7), we substitute Eqs. (C4)-(C7), then Eqs. (C8)-(C11), and symmetrize: $\bar{S}_{Y_{\text {hom }}}(\omega)=$ $\frac{S_{Y_{\text {hom }}}(\omega)+S_{Y_{\text {hom }}}(-\omega)}{2}$.

\section{APPENDIX D: PARAMETERS}

Figure 1(a): Parameters correspond to the experiment in [26]: mechanical frequency $\omega_{M} / 2 \pi=1.53 \mathrm{MHz}$, detuning of the control and probe modes $\Delta=-\omega_{M}$ and $\Delta_{p} / 2 \pi=$ $-21 \mathrm{kHz}$ with $g_{1}^{(c)} / 2 \pi=3.5 \times 10^{4} \mathrm{~Hz}$ for control beam and $g_{1}^{(p)} / 2 \pi=3.5 \times 10^{5} \mathrm{~Hz}$ for probe beam, cavity damping $\kappa / 2 \pi=0.85 \mathrm{MHz}$, mechanical damping $\gamma / 2 \pi=0.22 \mathrm{~Hz}$, and bath temperature $T=4.6 \mathrm{~K}$.

Figure 1(b): Parameters were taken from Refs. [19,33]: $\omega_{M} / 2 \pi=50 \mathrm{kHz}, \Delta=-\omega_{M}, \Delta_{p}=0, \bar{g}_{1}^{(c)} / 2 \pi=5 \mathrm{kHz}$, $\bar{g}_{1}^{(p)} / 2 \pi=50 \mathrm{kHz}, \omega_{d} / 2 \pi=1500 \mathrm{~Hz}, \kappa / 2 \pi=100 \mathrm{kHz}$, $T=300 \mathrm{~K}$, and $\gamma=0$ to isolate the quantum back-action contribution.

Figures 2 and 3: Parameters are the same as in Fig. 1(a).

Figure 4: Parameters are similar to Ref. [6] $\left(g_{1}^{(c)}=g_{1}^{(p)}=0\right)$ : $\omega_{M}=5 \kappa, g_{2}=\kappa / 2, \gamma=\kappa / 10$, and $\kappa / 2 \pi=0.85 \mathrm{MHz}$.
[1] M. Aspelmeyer, T. J. Kippenberg, and F. Marquardt, Rev. Mod. Phys. 86, 1391 (2014).

[2] A. A. Clerk, M. H. Devoret, S. M. Girvin, F. Marquardt, and R. J. Schoelkopf, Rev. Mod. Phys. 82, 1155 (2010).

[3] W. P. Bowen and G. J. Milburn, Quantum Optomechanics (CRC Press, Boca Raton, FL, 2016).

[4] J. D. Thompson, B. M. Zwickl, A. M. Jayich, F. Marquardt, S. M. Girvin, and J. G. E. Harris, Nature (London) 452, 72 (2008).

[5] D. Lee, M. Underwood, D. Mason, A. B. Shkarin, S. W. Hoch and J. G. E. Harris, Nat. Commun. 6, 6232 (2015).

[6] A. Nunnenkamp, K. Børkje, J. G. E. Harris, and S. M. Girvin, Phys. Rev. A 82, 021806(R) (2010).

[7] K. Børkje, A. Nunnenkamp, J. D. Teufel, and S. M. Girvin, Phys. Rev. Lett. 111, 053603 (2013).

[8] A. Kronwald and F. Marquardt, Phys. Rev. Lett. 111, 133601 (2013).

[9] G. A. Brawley, M. R. Vanner, P. E. Larsen, S. Schmid, A. Boisen, and W. P. Bowen, Nat. Commun. 7, 10988 (2016).

[10] R. Leijssen, G. R. L. Gala, L. Freisem, J. T. Muhonen, and E. Verhagen, Nat. Commun. 8, 16024 (2017).

[11] V. Sudhir, R. Schilling, S. A. Fedorov, H. Schütz, D. J. Wilson, and T. J. Kippenberg, Phys. Rev. X 7, 031055 (2017).

[12] T. P. Purdy, K. E. Grutter, K. Srinivasan, and J. M. Taylor, Science 356, 1265 (2017).
[13] J. Millen, P. Z. G. Fonseca, T. Mavrogordatos, T. S. Monteiro, and P. F. Barker, Phys. Rev. Lett. 114, 123602 (2015).

[14] P. Z. G. Fonseca, E. B. Aranas, J. Millen, T. S. Monteiro, and P. F. Barker, Phys. Rev. Lett. 117, 173602 (2016).

[15] J. Gieseler, L. Novotny, and R. Quidant, Nat. Phys. 9, 806 (2013).

[16] M. Rashid, M. Toroš, A. Setter, and H. Ulbricht, arXiv:1805.08042.

[17] A. Pontin, J. E. Lang, A. Chowdhury, P. Vezio, F. Marino, B. Morana, E. Serra, F. Marin, and T. S. Monteiro, Phys. Rev. Lett. 120, 020503 (2018).

[18] A. Setter, M. Toroš, J. F. Ralph, and H. Ulbricht, Phys. Rev. A 97, 033822 (2018).

[19] E. B. Aranas, M. J. Akram, D. Malz, and T. S. Monteiro, Phys. Rev. A 96, 063836 (2017).

[20] M. Toroš, M. Rashid, and H. Ulbricht, Phys. Rev. A 98, 053803 (2018).

[21] Downloadable from www.xmds.org.

[22] A. H. Safavi-Naeini, J. Chan, J. T. Hill, T. P. M. Alegre, A. Krause, and O. Painter, Phys. Rev. Lett. 108, 033602 (2012).

[23] F. Y. Khalili, H. Miao, H. Yang, A. H. Safavi-Naeini, O. Painter, and Y. Chen, Phys. Rev. A 86, 033840 (2012).

[24] D. W. C. Brooks, T. Botter, S. Schreppler, T. P. Purdy, N. Brahms, and D. M. Stamper-Kurn, Nature (London) 488, 476 (2012). 
[25] A. H. Safavi-Naeini, S. Gröblacher, J. T. Hill, J. Chan, M. Aspelmeyer, and O. Painter, Nature (London) 500, 185 (2013).

[26] T. P. Purdy, P. L. Yu, R. W. Peterson, N. S. Kampel, and C. A. Regal, Phys. Rev. X 3, 031012 (2013).

[27] A. Pontin, C. Biancofiore, E. Serra, A. Borrielli, F. S. Cataliotti, F. Marino, G. A. Prodi, M. Bonaldi, F. Marin, and D. Vitali, Phys. Rev. A 89, 033810 (2014).

[28] V. Sudhir, D. J. Wilson, R. Schilling, H. Schütz, S. A. Fedorov, A. H. Ghadimi, A. Nunnenkamp, and T. J. Kippenberg, Phys. Rev. X 7, 011001 (2017).

[29] R. W. Peterson, T. P. Purdy, N. S. Kampel, R. W. Andrews, P.-L. Yu, K. W. Lehnert, and C. A. Regal, Phys. Rev. Lett. 116, 063601 (2016).

[30] A. H. Safavi-Naeini, J. Chan, J. T. Hill, S. Gröblacher, H. Miao, Y. Chen, M. Aspelmeyer, and O. Painter, New J. Phys. 15, 035007 (2013).

[31] C. Fabre, M. Pinard, S. Bourzeix, A. Heidmann, E. Giacobino, and S. Reynaud, Phys. Rev. A 49, 1337 (1994).
[32] T. K. Paraïso, M. Kalaee, L. Zang, H. Pfeifer, F. Marquardt, and O. Painter, Phys. Rev. X 5, 041024 (2015).

[33] E. B. Aranas, P. Z. G. Fonseca, P. F. Barker, and T. S. Monteiro, New J. Phys. 18, 113021 (2016).

[34] G. R. Dennis, J. J. Hope, and M. T. Johnsson, Comput. Phys. Commun. 184, 201 (2013).

[35] J. Wilkie, Phys. Rev. E 70, 017701 (2004).

[36] L. F. Buchmann, S. Schreppler, J. Kohler, N. Spethmann, and D. M. Stamper-Kurn, Phys. Rev. Lett. 117, 030801 (2016).

[37] C. F. Ockeloen-Korppi, E. Damskägg, G. S. Paraoanu, F. Massel, and M. A. Sillanpää, arXiv:1809.02396.

[38] A. J. Weinstein, C. U. Lei, E. E. Wollman, J. Suh, A. Metelmann, A. A. Clerk, and K. C. Schwab, Phys. Rev. X 4, 041003 (2014).

[39] K. Børke, Phys. Rev. A 94, 043816 (2016).

[40] F. Marquardt, J. P. Chen, A. A. Clerk, and S. M. Girvin, Phys. Rev. Lett. 99, 093902 (2007). 